BMJ Open

Diabetes

Research

\& Care

\title{
Zinc transporter 8 autoantibody testing requires age-related cut-offs
}

\author{
Sian Louise Grace $\mathbb{0},{ }^{1}$ Angela Cooper, ${ }^{2}$ Angus G Jones,,${ }^{1,3}$ \\ Timothy James McDonald (D) 1,2
}

To cite: Grace SL, Cooper A, Jones AG, et al. Zinc transporter 8 autoantibody testing requires age-related cut-offs. BMJ Open Diab Res Care 2021;9:e002296. doi:10.1136/ bmjdrc-2021-002296

- Additional supplemental material is published online only. To view, please visit the journal online (http://dx.doi. org/10.1136/bmjdrc-2021002296)

Received 31 March 2021 Accepted 17 July 2021

Check for updates

(C) Author(s) (or their employer(s)) 2021. Re-use permitted under CC BY-NC. No commercial re-use. See rights and permissions. Published by BMJ.

${ }^{1}$ The Institute of Biomedical \& Clinical Science, University of Exeter, Exeter, UK

${ }^{2}$ Academic Department of Clinical Biochemistry, Royal Devon and Exeter NHS Foundation Trust, Exeter, UK ${ }^{3}$ Macleod Diabetes and Endocrine Centre, Royal Devon and Exeter NHS Foundation Trust, Exeter, UK

Correspondence to Professor Timothy James McDonald;

timothy.mcdonald@nhs.net

\section{ABSTRACT}

Introduction Zinc transporter 8 autoantibodies (ZnT8A)

are biomarkers of beta cell autoimmunity in type 1 diabetes that have become more widely available to clinicians in recent years. Robust control populationdefined thresholds are essential to ensure high clinical specificity in islet autoantibody testing. We aimed to determine the optimal cut-offs for ZnT8A testing. Research design and methods 97.5 th and 99th centile cut-offs were determined using residual clinical sera from 1559 controls aged between 0 and 83 years with no history of diabetes and a hemoglobin A1c level of less than $6.0 \%(<42 \mathrm{mmol} / \mathrm{mol})$. ZnT8A were measured by ELISA (RSR, Cardiff, UK) on a Dynex DS2 ELISA robot (Dynex, Preston, UK). We assessed the impact of age-related cutoffs in comparison with the manufacturer's recommended threshold in a mixed cohort of young-onset (<age 30) diabetes (UNITED study (Using pharmacogeNetics to Improve Treatment in Early-onset Diabetes), $n=145$ ). Results Using the manufacturer's limit of detection, 6 WHO U/mL, $16.2 \%$ of people in the control cohort had detectable levels of ZnT8A and those who had detectable ZnT8A were much more likely to be younger $(p<0.0001)$. The 97.5th and 99th centile thresholds were substantially higher in younger participants: 18 and $127 \mathrm{WHO} \mathrm{U} / \mathrm{mL}$ (tested under 30 years) in comparison with 9 and 21 WHO U/mL (tested 30 years and over). In the UNITED cohort some of those found to be ZnT8A-positive by the manufacturer's threshold but negative using the appropriate $99 \%$ centile cut-off (127 WHO U/mL) displayed characteristics suggestive of type 2 diabetes.

Conclusions Age-related thresholds are needed for ZnT8A testing. In those aged $<30$ years, use of manufacturers' recommended cut-offs may result in low test specificity and potentially high rates of false positive test results in patients who do not have autoimmune diabetes.

\section{INTRODUCTION}

Islet autoantibodies, including glutamate decarboxylase (GADA), islet antigen-2 (IA2A) and insulin, have traditionally been used as biomarkers of the autoimmune attack occurring in type 1 diabetes (T1D). The presence of islet autoantibodies is useful in differentiating T1D from other forms of diabetes and is used in research to stratify the risk of progression to T1D in cohort studies. ${ }^{12}$ Zinc transporter 8 autoantibodies (ZnT8A) are

\section{Significance of this study}

What is already known about this subject?

- Zinc transporter 8 autoantibodies (ZnT8A) are increasingly used in clinical practice for classification of diabetes and for prediction of type 1 diabetes in a research setting.

- Thresholds of positivity have not yet been robustly defined and the impact of age on thresholds has not been examined.

What are the new findings?

- Healthy controls, with no history of diabetes and aged under 30 years, are more likely to have higher levels of detectable ZnT8A.

- The 97.5th and 99th centile cut-offs were significantly higher in those tested under 30 years in comparison with those tested over 30 years.

How might these results change the focus of research or clinical practice?

- Clinical laboratories need to establish robust agespecific thresholds for ZnT8A testing, as without these, false positive results are likely to be common in young people with diabetes of non-autoimmune etiology.

the most recently characterized islet autoantibody target of T1D autoimmunity, found in $66 \%-80 \%$ of Caucasian patients at diagnosis and detectable in approximately $26 \%$ of individuals with T1D previously categorized as autoantibody-negative. ${ }^{3}$ In those diagnosed young, ZnT8A have been found to be associated with a more acute onset of disease and a greater probability of ketoacidosis at presentation. ${ }^{4}$ ZnT8A have also been found to help exclude maturity onset diabetes of the young (MODY), identifying an additional $18 \%$ of probable T1D (in comparison with GADA and IA-2A autoantibodies alone) in individuals with significant endogenous insulin secretion. ${ }^{5}$ ZnT8A testing has become widely available in clinical practice.

The interpretation of islet autoantibodies requires robust and well-defined cut-offs to ensure high clinical specificity. Islet autoantibodies are found on a continuum of 
concentration and are often detectable at low levels in the population without diabetes. ${ }^{67}$ Therefore, islet autoantibody cut-offs are normally based on centiles of a control population. Usually, the 97.5 th or 99 th centile of a population without diabetes is used as a positive cutoff (specificity $97.5 \%$ or $99 \%$ ), depending on the specificity required for the clinical scenario. ${ }^{8-10}$ However, testing of sufficient numbers of controls without diabetes to accurately determine test threshold is expensive, and in practice the use of the limit of detection (LOD), or manufacturers' recommended cut-off, is common. ${ }^{11}$ The impact of age on optimal thresholds of ZnT8A positivity has not yet been assessed.

We aimed to define robust thresholds of positivity for ZnT8A testing in a European population using the RSR ZnT8A ELISA assay and assess whether these are affected by age of the participant tested.

\section{PARTICIPANTS AND METHODS \\ Study participants \\ Setting the threshold}

To establish an antibody titer cut-off for ZnT8A and assess the impact of age, residual sera from 1559 routine clinical samples of patients aged between 0 and 83 years of age without a clinical history of diabetes and a hemoglobin A1c level of less than $6.0 \%(<42 \mathrm{mmol} / \mathrm{mol})$ were analyzed at the Academic Department of Blood Sciences, Royal Devon and Exeter Hospital (Exeter, UK).

\section{Assessment of threshold validity}

Threshold validity and the effects of age-related cut-offs were assessed on 145 patients with young-onset diabetes (111 diagnosed with T1D, 31 with type 2 diabetes and 3 with another diabetes type). Patients were identified from the UNITED (Using pharmacogeNetics to Improve Treatment in Early-onset Diabetes) cohort, ${ }^{12}$ an unselected cross-sectional cohort of participants diagnosed with diabetes less than 30 years, but recruited before the age of 50 years. All included participants had been tested for ZnT8A (online supplemental table 1).

\section{ZnT8A analysis}

ZnT8A were analyzed using an ELISA commercial kit (RSR, Cardiff, UK) on a Dynex DS2 ELISA robot (Dynex, Preston, UK). The RSR ZnT8A ELISA is capable of detecting and quantifying autoantibodies specific to R325 or to W325 polymorphic variants of ZnT8A. This laboratory participates in the islet autoantibody standardization program (IASP), an external blinded quality assurance program for islet autoantibodies. ${ }^{13}$ The assay LOD is $6 \mathrm{U} / \mathrm{mL}$ and the manufacturer's recommended cut-off for positivity $\geq 15 \mathrm{U} / \mathrm{mL}$.

\section{Data analysis}

The 5-year rolling average percentage with detectable ZnT8A and ZnT8A median titer were plotted (figure 1). The cut-offs were defined by using the 97.5th and 99th centiles. The specificity of the manufacturer's LOD was

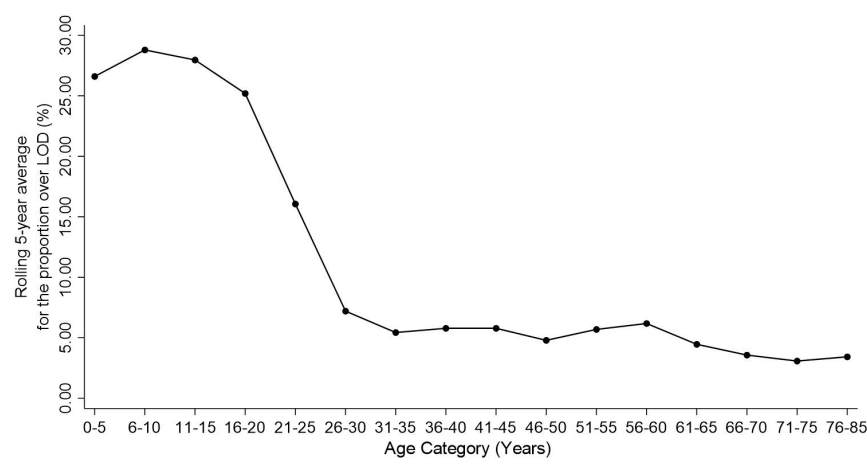

Figure 1 Line graph of the 5-year rolling average of the percentage of those with detectable ZnT8A. The rolling average shows a drop to consistently $<10 \%$ with detectable $\mathrm{ZnT8A}$ after the age of 30 years. LOD $\geq 6 \mathrm{U} / \mathrm{mL}$. LOD, limit of detection; ZnT8A, zinc transporter 8 autoantibodies.

assessed by applying it to the overall control cohort, conducting a subanalysis of each age group (less than 30 years and 30 years and over) and evaluating by the proportion positive. Fisher's exact test was used to compare groups above and below the LOD.

To assess the validity of the newly defined age-related cut-offs, the 99th centile cut-offs were applied to reduce the potential misclassification of patients with MODY, retrospectively to the UNITED cohort. ${ }^{5}$ In those tested under 30, we assessed the characteristics of participants who were positive for ZnT8A using the manufacturer's recommended cut-off $(15 \mathrm{U} / \mathrm{mL})$ but negative for ZnT8A using an appropriate (99th centile) cut-off based from the control population of comparable age.

All statistical analyses were carried out using Stata/SE V.16.0, and figure 2 was drawn with GraphPad Prism Software V.8.

\section{RESULTS}

Detectable $\mathrm{ZnT}$ TA levels in the control population are common in those aged under 30 years

Using the manufacturer's reported LOD, $6 \mathrm{U} / \mathrm{mL}, 16.2 \%$ (95\% CI 0.14 to 0.18 ) of participants in the control cohort had detectable ZnT8A. The 5-year rolling average of $\%$ detectable was plotted, and a marked decline in $\%$ detectable in early adulthood with stable low levels of detectable ZnT8A after age 30 was observed (figure 1). Therefore we split the control cohort into those tested under and over 30 years. The prevalence of detectable ZnT8A was strongly related to age: in those aged under 30 years, $21 \%(\mathrm{n}=229,79 \%$ specificity, $95 \% \mathrm{CI} 0.19$ to $0.24)$ had detectable ZnT8A; in contrast only $5 \%(\mathrm{n}=24$, $95 \%$ specificity, $95 \%$ CI 0.03 to 0.07 ) of those tested 30 years and over were over the LOD $(\mathrm{p}<0.0001)$.

\section{Appropriate cut-offs for ZnT8A testing widely vary by age}

For those tested under 30 years, the 97.5th and 99th centile cut-offs were $18 \mathrm{U} / \mathrm{mL}$ and $127 \mathrm{U} / \mathrm{mL}$, respectively. However, for those tested aged 30 years and over, the 97.5th and 99th centile cut-offs were $9 \mathrm{U} / \mathrm{mL}$ and $21 \mathrm{U} / \mathrm{mL}$ (figure 2). Therefore, to maintain assay 


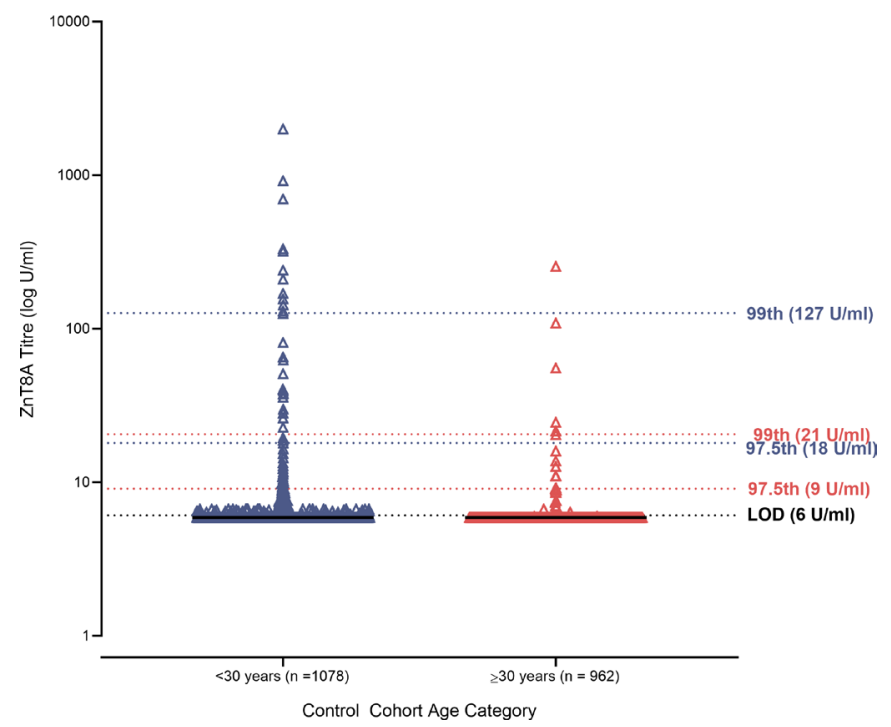

Figure 2 Plot of ZnT8A titers in healthy controls $(n=1559)$.

Dotted lines indicate the 97.5th and 99th percentiles for $<30$ years (purple; $n=1078$ ) and $\geq 30$ years (red; $n=481$ ). Black dotted line indicates the LOD $(6 \mathrm{U} / \mathrm{mL})$. Black solid lines indicate the median ZnT8A titer. LOD, limit of detection; ZnT8A, zinc transporter 8 autoantibodies.

specificity, those tested under the age of 30 years would need a higher cut-off in comparison with those tested aged 30 and over.

\section{Age-related cut-offs for ZnT8A testing reclassify patients as negative who have a more type 2 diabetic phenotype}

When the age-related 99th centile cut-offs of $127 \mathrm{U} /$ $\mathrm{mL}$ and $21 \mathrm{U} / \mathrm{mL}$ (increased from the manufacturer's cut-off, $15 \mathrm{U} / \mathrm{mL}$ ) were applied to the UNITED cohort retrospectively, 23 participants tested under 30 years were reclassified as ZnT8A-negative. Of these eight were now classified as autoantibody-negative and some exhibited a more type 2 diabetic phenotype (table 1 ). Three of eight were non-insulin-treated, and three of five had high urinary $\mathrm{C}$ peptide to creatinine ratio levels $(\geq 0.6 \mathrm{nmol} / \mathrm{L})$. Moreover, four of the patients were overweight $(\geq 25)$ and three were obese $(\geq 30)$. In contrast, in those who remained positive for ZnT8A, above the 99th centile age-related cut-off, $85 \%$ were positive for one or more additional islet autoantibodies (GADA or IA-2A) and all those who were single ZnT8A positive above the 99th centile were on insulin treatment.

\section{Age-specific thresholds for ZnT8A in the IASP increased specificity without loss of sensitivity}

When the 99th centile age-specific cut-offs described here were applied to a separate set of control samples $(n=140)$ as part of the IASP 2020 workshop, specificity was high $(98.9 \%)$, without loss of sensitivity $(74.0 \%)$. This is in comparison with a specificity of $97 \%$ and a sensitivity of $74 \%$ reported in the assay insert by the manufacturer when their $\geq 15 \mathrm{U} / \mathrm{mL}$ cut-off was used. ${ }^{14}$

\section{DISCUSSION}

This study has shown that detectable ZnT8A levels above the manufacturer's LOD are very common in a control population without diabetes, occurring in $21 \%$ of those tested under the age of 30 years and in $5 \%$ of those tested aged 30 and over. Where ZnT8A are detectable, titers are higher in younger individuals. Therefore using the same cut-off to define ZnT8A positivity for all ages could result in lower test specificity in young patients and potentially high rates of false positive test results. In our unselected population with young-onset diabetes, some of the participants with positive ZnT8A above the manufacturer's recommended cut-off but ZnT8A-negative using an

\begin{tabular}{|c|c|c|c|c|c|c|c|c|c|}
\hline $\begin{array}{l}\text { Patient } \\
\text { number }\end{array}$ & $\begin{array}{l}\text { Age of diagnosis } \\
\text { bracket } \\
\text { (years)* }^{*}\end{array}$ & $\begin{array}{l}\text { Diabetes } \\
\text { duration } \\
\text { (years) }\end{array}$ & $\begin{array}{l}\text { Current } \\
\text { treatment† }\end{array}$ & $\begin{array}{l}\text { Adjusted } \\
\text { BMI† }\end{array}$ & $\begin{array}{l}\text { HbA1c } \\
\text { (mmol/mol) } \\
(\%) \dagger\end{array}$ & $\begin{array}{l}\text { UCPCR } \\
\text { (nmol/mmol) }\end{array}$ & $\begin{array}{l}\text { Duration } \\
\text { at UCPCR } \\
\text { (years) }\end{array}$ & $\begin{array}{l}\text { ZnT8A } \\
\text { titer } \\
(\mathrm{U} / \mathrm{mL})\end{array}$ & $\begin{array}{l}\text { Duration at } \\
\text { ZnT8A } \\
\text { (years) }\end{array}$ \\
\hline 1 & $10-15$ & 3.8 & Insulin & 28.3 & $48(6.5)$ & 0.79 & 3.8 & 56.6 & 4.2 \\
\hline 2 & $10-15$ & 14.8 & Insulin & 24.5 & $48(6.5)$ & 0.29 & 14.8 & 54.1 & 15.1 \\
\hline 3 & $15-20$ & 0.2 & Insulin & 46.7 & 107 (11.9) & 2.02 & 0.2 & 52.4 & 0.1 \\
\hline 4 & $15-20$ & 5.9 & Insulin & 21.0 & $86(10)$ & 0.21 & 5.9 & 35.9 & 6.0 \\
\hline 5 & $25-30$ & 1.0 & Tablet & 47.5 & $53(7)$ & - & - & 32.7 & 1.0 \\
\hline 6 & $20-25$ & 1.2 & Insulin & 22.0 & $43(6.1)$ & 0.98 & 3.0 & 26.6 & 3.1 \\
\hline 7 & $25-30$ & 0.9 & Tablet & - & $40(5.8)$ & - & - & 24.9 & 1.0 \\
\hline 8 & $25-30$ & 0.2 & Tablet & 32.5 & $115(12.7)$ & - & - & 17.2 & 0.0 \\
\hline
\end{tabular}

Inclusion criteria were now negative for ZnT8A with new 99th centile cut-off and negative for glutamate decarboxylase and islet antigen-2 autoantibodies.

- indicates missing data.

For UCPCR, a result $\geq 0.6$ indicates substantial endogenous insulin secretion.

ZnT8A titer: level of units of ZnT8A present in the serum.

${ }^{*}$ For reasons of patient anonymity.

†At latest follow-up.

BMI, body mass index; HbA1c, hemoglobin A1c; UCPCR, urinary C peptide to creatinine ratio; UNITED, Using pharmacogeNetics to Improve

Treatment in Early-onset Diabetes; ZnT8A, zinc transporter 8 autoantibodies. 
appropriate population-based (99th centile) threshold had the clinical characteristics of type 2 diabetes.

The 99th age-specific cut-offs improved performance of this assay in IASP, increasing specificity without loss of sensitivity (98.9\% and $74.0 \%$, respectively). The average specificity and sensitivity were $94.4 \%$ and $64.1 \%$ for this assay across the other laboratories submitting results, ${ }^{15}$ therefore increasing confidence in the use of agerestricted thresholds for this assay.

The mechanisms that result in increased background prevalence and titer of ZnT8A in the young healthy control cohort are unknown. In healthy people we may speculate that it could be due to differences in immune system maturity or cross-reactivity to an infection mode commonly seen in the young, for example, crossreactivity between ZnT8A and an antigen expressed by Mycobacterium avium subspecies paratuberculosis, which has been described. ${ }^{16}$ Another possibility for the higher titer and prevalence of ZnT8A in those tested $<30$ years in the control cohort is that there is a potential enrichment of individuals in the prodrome stage of T1D. However, the overall life prevalence of T1D is less than $0.3 \%$ (with approximately half of cases occurring after age 30$)^{17}$ and therefore will be contributing only a very small amount to the difference between $<30$ and $>30$ cohorts $(\sim 0.15 \%)$. To our knowledge, this is one of the first studies to show age effects of ZnT8A on thresholds of positivity. A previous study by Vermeulen $e t a l^{\hat{l}}$ reported using age-restricted cut-offs for positivity for their liquid-phase radiobinding assay to detect ZnT8A. Their age cut-offs differed from ours (0-14 years and 15-39 years) and their control population was considerably smaller $(n=761)$, with only those aged up to 39 assessed.

Due to the nature of the control cohort, the findings of this study are limited to one population which is predominantly of European descent. In addition, this study only used one assay type for ZnT8A; however, the RSR ELISA is used by many clinical laboratories since it was distributed and validated in 2013. ${ }^{18-20}$ Differences in ZnT8A prevalence have been reported in non-white ethnic patients with diabetes; defining appropriate reference ranges for different ethnicities and for other assays will be important areas for future research. ${ }^{21}{ }^{22} \mathrm{~A}$ further limitation is that although this is a large cohort, far in excess of what has been previously reported, ${ }^{9} 1923$ the sample size is still insufficient to do more than visually assess an optimal age cut-off. Therefore, an even larger sample size would be needed to give greater detail on optimal test cut-offs for different age groups.

High clinical specificity of islet autoantibody tests is of particular importance in the setting of low prior prevalence, such as the case of prediction of T1D in the general population or the diagnosis of autoimmune diabetes in older adults. ${ }^{17}$ By setting robust and well-defined cutoffs for each assay based on appropriate control populations, this will ensure high assay specificity and reduce potentially high rates of false positives in those with nonautoimmune diabetes. This is of increasing importance for studies into the prediction of T1D due to the recent progressions in intervention therapy research ${ }^{24}$ and in differentiating autoimmune from other forms of diabetes. ${ }^{25}$ We have shown potential misclassification of diabetes types due to the use of manufacturers' recommended cut-off. This is likely to affect previous work where reported prevalence of $\mathrm{ZnT}$ 8A has varied and manufacturers' guidelines of cut-offs have been used instead of a robust population-defined threshold.

\section{CONCLUSION}

In those aged $<30$ years, a higher age-related threshold is likely to be needed for ZnT8A testing to prevent low test specificity and potentially high rates of false positive test results in patients who do not have autoimmune diabetes.

Acknowledgements We would like to thank the UNITED study participants for their participation in the study. We would also like to thank Dr Beverley Shields for her advice in relation to the statistical analysis.

Contributors SLG, AC, AGJ and TJM conceived the idea and designed the study. $A C$ and TJM collected the data. SLG analyzed the data with assistance from AC, AGJ and TJM. SLG drafted the manuscript with assistance from AGJ and TJM. All authors critically revised the manuscript and approved the final version. TJM is the guarantor of this work and, as such, has full access to all the data in the study and takes responsibility for the integrity of the data and the accuracy of the data analysis.

Funding SLG is supported by a PhD Studentship funded by an Expanding Excellence in England award from Research England. AGJ is supported by an NIHR Clinician Scientist Award (CS-2015-15-018). TJM is a National Institute for Health Research Senior Clinical Senior Lecturer. The views given in this article do not necessarily represent those of the National Institute for Health Research, the National Health Service, the Department of Health and Social Care, or Research England.

Competing interests None declared.

Patient consent for publication Not required.

Ethics approval Anonymized residual clinical material was used to establish the cut-offs. This is in line with HTA guidance on use of residual clinical material and was approved by the Royal Devon and Exeter Pathology Governance Group. Patients consented samples as part of the biomarker screening pathway (the Using pharmacogeNetics to Improve Treatment in Early-onset Diabetes (UNITED) study, ClinicalTrials.gov NCT01238380, REC 10/H0106/63).

Provenance and peer review Not commissioned; externally peer reviewed.

Data availability statement Data are available upon reasonable request.

Supplemental material This content has been supplied by the author(s). It has not been vetted by BMJ Publishing Group Limited (BMJ) and may not have been peer-reviewed. Any opinions or recommendations discussed are solely those of the author(s) and are not endorsed by BMJ. BMJ disclaims all liability and responsibility arising from any reliance placed on the content. Where the content includes any translated material, BMJ does not warrant the accuracy and reliability of the translations (including but not limited to local regulations, clinical guidelines, terminology, drug names and drug dosages), and is not responsible for any error and/or omissions arising from translation and adaptation or otherwise.

Open access This is an open access article distributed in accordance with the Creative Commons Attribution Non Commercial (CC BY-NC 4.0) license, which permits others to distribute, remix, adapt, build upon this work non-commercially, and license their derivative works on different terms, provided the original work is properly cited, appropriate credit is given, any changes made indicated, and the use is non-commercial. See: http://creativecommons.org/licenses/by-nc/4.0/.

ORCID IDS

Sian Louise Grace http://orcid.org/0000-0001-6925-9780

Timothy James McDonald http://orcid.org/0000-0003-3559-6660 
REFERENCES

1 Ziegler AG, Rewers M, Simell O, et al. Seroconversion to multiple islet autoantibodies and risk of progression to diabetes in children. JAMA 2013;309:2473-9.

2 Garnier L, Marchand L, Benoit M, et al. Screening of ZnT8 autoantibodies in the diagnosis of autoimmune diabetes in a large French cohort. Clinica Chimica Acta 2018;478:162-5.

3 Wenzlau JM, Juhl K, Yu L, et al. The cation efflux transporter ZnT8 (Slc30A8) is a major autoantigen in human type 1 diabetes. Proc Natl Acad Sci U S A 2007;104:17040-5.

4 Niechciał E, Rogowicz-Frontczak A, Piłaciński S, et al. Autoantibodies against zinc transporter 8 are related to age and metabolic state in patients with newly diagnosed autoimmune diabetes. Acta Diabetol 2018;55:287-94.

5 Patel KA, Weedon MN, Shields BM, et al. Zinc transporter 8 autoantibodies (ZnT8A) and a type 1 diabetes genetic risk score can exclude individuals with type 1 diabetes from inappropriate genetic testing for monogenic diabetes. Diabetes Care 2019;42:e16-17.

6 Schlosser M, Strebelow M, Rjasanowski I, et al. Prevalence of diabetes-associated autoantibodies in schoolchildren: the Karlsburg type 1 diabetes risk study. Ann N Y Acad Sci 2004;1037:114-7.

7 Vermeulen I, Weets I, Asanghanwa M, et al. Contribution of antibodies against IA-2 $\beta$ and zinc transporter 8 to classification of diabetes diagnosed under 40 years of age. Diabetes Care 2011;34:1760-5

8 Bingley PJ. Clinical applications of diabetes antibody testing. J Clin Endocrinol Metab 2010;95:25-33.

9 Kawasaki E, Nakamura K, Kuriya G, et al. Zinc transporter 8 autoantibodies in fulminant, acute-onset, and slow-onset patients with type 1 diabetes. Diabetes Metab Res Rev 2011;27:895-8.

10 Andersen MK, Härkönen T, Forsblom C, et al. Zinc transporter type 8 autoantibodies (ZnT8A): prevalence and phenotypic associations in latent autoimmune diabetes patients and patients with adult onset type 1 diabetes. Autoimmunity 2013;46:251-8.

11 Dunseath G, Ananieva-Jordanova R, Coles R, et al. Bridging-type enzyme-linked immunoassay for zinc transporter 8 autoantibody measurements in adult patients with diabetes mellitus. Clin Chim Acta 2015;447:90-5.

12 Shields BM, Shepherd M, Hudson M, et al. Population-Based assessment of a biomarker-based screening pathway to aid diagnosis of monogenic diabetes in young-onset patients. Diabetes Care 2017:40:1017-25.
13 Lampasona V, Schlosser M, Mueller PW, et al. Diabetes antibody standardization program: first proficiency evaluation of assays for autoantibodies to zinc transporter 8. Clin Chem 2011;57:1693-702.

14 ELISA RSRTM ZnT8 AbTM Technical Information. Cardiff (U.K.): RSR; 2015.

15 Immunology of Diabetes Society. IASP 2018 Cummulative performance summary Univeristy of Florida; 2018.

16 Niegowska M, Rapini N, Piccinini S, et al. Type 1 diabetes atrisk children highly recognize Mycobacterium avium subspecies paratuberculosis epitopes homologous to human Znt8 and proinsulin. Sci Rep 2016;6:22266.

17 Thomas NJ, Jones SE, Weedon MN, et al. Frequency and phenotype of type 1 diabetes in the first six decades of life: a cross-sectional, genetically stratified survival analysis from UK Biobank. Lancet Diabetes Endocrinol 2018;6:122-9.

18 Hörber S, Achenbach P, Schleicher E, et al. Harmonization of immunoassays for biomarkers in diabetes mellitus. Biotechnol Adv 2020;39:107359

19 Kawasaki E, Tanaka M, Miwa M, et al. Novel enzyme-linked immunosorbent assay for bivalent ZnT8 autoantibodies. Acta Diabetol 2014;51:429-34.

20 Wasserfall C, Montgomery E, Yu L, et al. Validation of a rapid type 1 diabetes autoantibody screening assay for community-based screening of organ donors to identify subjects at increased risk for the disease. Clin Exp Immunol 2016;185:33-41.

21 Yang L, Luo S, Huang G, et al. The diagnostic value of zinc transporter 8 autoantibody (ZnT8A) for type 1 diabetes in Chinese. Diabetes Metab Res Rev 2010;26:579-84.

22 Vaziri-Sani F, Oak S, Radtke J, et al. ZnT8 autoantibody titers in type 1 diabetes patients decline rapidly after clinical onset. Autoimmunity 2010;43:598-606.

23 Bizzarri C, Giannone G, Benevento D, et al. ZnT8 antibodies in patients with cystic fibrosis: an expression of secondary beta-cel damage? J Cyst Fibros 2013;12:803-5.

24 Herold KC, Bundy BN, Long SA, et al. An anti-CD3 antibody, Teplizumab, in relatives at risk for type 1 diabetes. $N$ Engl J Med 2019;381:603-13.

25 Thomas NJ, Lynam AL, Hill AV, et al. Type 1 diabetes defined by severe insulin deficiency occurs after 30 years of age and is commonly treated as type 2 diabetes. Diabetologia 2019;62:1167-72. 